OPEN ACCESS

Edited by:

Yong Cui,

China-Japan Friendship

Hospital, China

Reviewed by:

ZhiQiang Yin,

Nanjing Medical University, China

Irina Khamaganova,

Pirogov Russian National Research

Medical University, Russia

*Correspondence:

Min Gao

gm/hx@163.com

Specialty section:

This article was submitted to

Dermatology,

a section of the journal

Frontiers in Medicine

Received: 04 January 2021 Accepted: 03 February 2021 Published: 24 February 2021

Citation:

Feng L, Lin Y, Wang L, Chen $H$, Gao M, Liu H and Yang H (2021) Imaging of Vulva Syringoma With Reflectance Confocal Microscopy. Front. Med. 8:649438 doi: 10.3389/fmed.2021.649438

\section{Imaging of Vulva Syringoma With Reflectance Confocal Microscopy}

\author{
Lin Feng ${ }^{1}$, Yan Lin ${ }^{2}$, Leilei Wang ${ }^{3}$, Hongxiao Chen ${ }^{4}$, Min Gao ${ }^{3 *}$, Huaxu Liu $^{2}$ and \\ Hongyu Yang ${ }^{5}$
}

${ }^{1}$ Chongqing Hospital of Traditional Chinese Medicine, Chongqing, China, ${ }^{2}$ Shandong Provincial Institute of Dermatology and Venereology, Shandong First Medical University \& Shandong Academy of Medical Sciences, Jinan, China, ${ }^{3}$ Shandong Cancer Hospital and Institute, Shandong First Medical University \& Shandong Academy of Medical Sciences, Jinan, China, ${ }^{4}$ Linyi People's Hospital, Linyi, China, ${ }^{5}$ Department of Pathology, St. Vincent Evansville Medical Center, Evansville, IN, United States

Objectives: To investigate the application of reflectance confocal microscopy (RCM) imaging in diagnosis of vulva syringoma.

Methods: Patients with lesions suspicious of syringoma on vulva were enrolled in the study. After informed consent was taken, the lesions were photographed and imaged with RCM. The features of the lesion in confocal images were then analyzed and compared with the biopsy findings for histology correlation.

Results: Eleven cases in total were included in the study. The typical RCM features observed in syringoma are the presence of round to oval high refractive, and relatively monomorphous mass of varying sizes in the superficial and middle dermis, usually surrounded with 1-2 layers of light-dark line structures, which were further confirmed by histological evaluation. Ten cases showed classic features of syringoma and 1 case exhibited milia in RCM images.

Conclusions: Syringoma has distinct features in RCM imaging, which correlates well with histological findings, highlighting the potential role of RCM in the diagnosis and differential diagnosis of vulva syringoma.

Keywords: in vivo imaging, reflectance confocal microcopy, vulva, syringoma, histology, differential diaenosis

\section{INTRODUCTION}

Syringoma is a benign sweat gland tumor derived from eccrine ducts and it occurs predominantly in women at puberty or later in life (1). The typical lesions of syringoma present as multiple, small, firm, skin-colored papules, usually $1-3 \mathrm{~mm}$ in diameter, and symmetrically distributed on the periorbital region $(1,2)$. Although the most common site of localized involvement is periorbital, syringoma in other areas have also been reported, including the vulva, penis, palms, scalp, and axillae $(2,3)$. The classification criterion of syringoma proposed by Friedman and Butler (4) was based on clinical features and consists of 4 variants: localized, familial (5), a form associated with Down Syndrome (DS), and a generalized variant, including multiple and eruptive syringoma (6).

The characteristics of lesions on the eyelids and forehead can be easily identified, while lesions in other areas especially on the vulva were not easily identifiable and the definitive diagnosis was usually made by histological examination. However, the invasive biopsy procedure for histology analysis reduced the compliance of the patients with suspicious vulva lesions, therefore developing a 
non-invasive skin imaging modality in the diagnosis and differential diagnosis of syringoma seems more appealing to potential patients.

The reflectance confocal microscopy (RCM) is a non-invasive skin imaging modality with high "cellular" resolution (7), which could compare the cellular changes of epidermis and superficial dermis in vivo in real time, and its accuracy is comparable with the histological evaluation of syringoma. In this study, we investigated the role of RCM in the diagnosis of vulva syringoma.

\section{PATIENTS AND METHODS}

The study had been approved by Ethics Committee of Shandong Cancer Hospital and Institute and was conducted from 2018 to 2019. Cases with lesions suspicious of syringoma on vulva (Figures 1a, 2a, 3a, 4a) were enrolled in the study. After written informed consent was signed, the lesions were photographed and imaged by a commercially available, reflectance mode confocal microscope (Vivascope 1500; Caliber Imaging \& Diagnostics,
Inc. formerly Lucid, Inc., Rochester, NY, USA). The captured horizontal images in a $500 \times 500 \mu \mathrm{m}$ field, and viva-block image of $3 \times 3 \mathrm{~mm}$ at different layers were obtained. A detailed description of the technique and the device has been published previously $(7,8)$. After imaging with RCM, the same lesions were biopsied and fixed in phosphate-buffered neutral formalin, embedded in paraffin, and stained with hematoxylin-eosin (HE), then analyzed using an optical microscope to investigate the accuracy of RCM imaging.

\section{RESULTS}

A total of 11 female adults were enrolled in the study (Table 1). The average age of the cases was 30.5 years old, and the average duration of the lesion was 17 months.

On clinical examination, multiple small, light brown papules are scattered in the vulva area (Figures 1a, 2a, 3a, 4a), or in a "beaded" distribution along the outer margin of labia Majora. The main characteristics of lesions from the patients observed
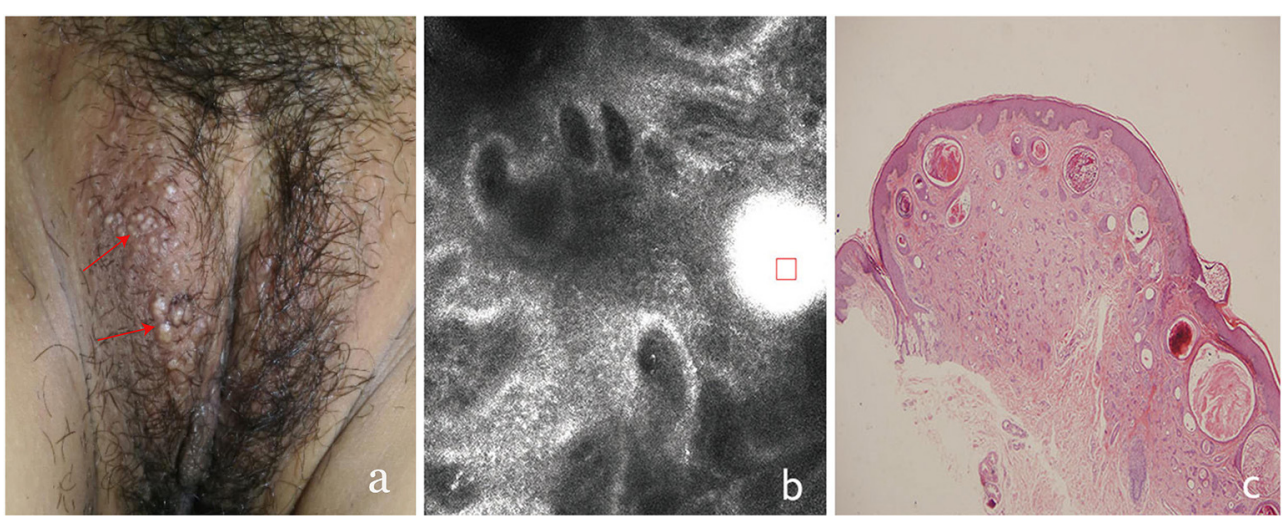

FIGURE 1 | The clinical presentation, RCM findings and histology of vulva syringoma (case 1). (a), the clinical image of case 1, (b), the RCM image findings; (c), the histology findings. The presence of round to oval high refractive, and relatively monomorphous mass of varing sizes (the red square)was prensent in the superficial dermis, with 1-2 layers of surrounding light-dark line structure (red arrowhead) in (b).
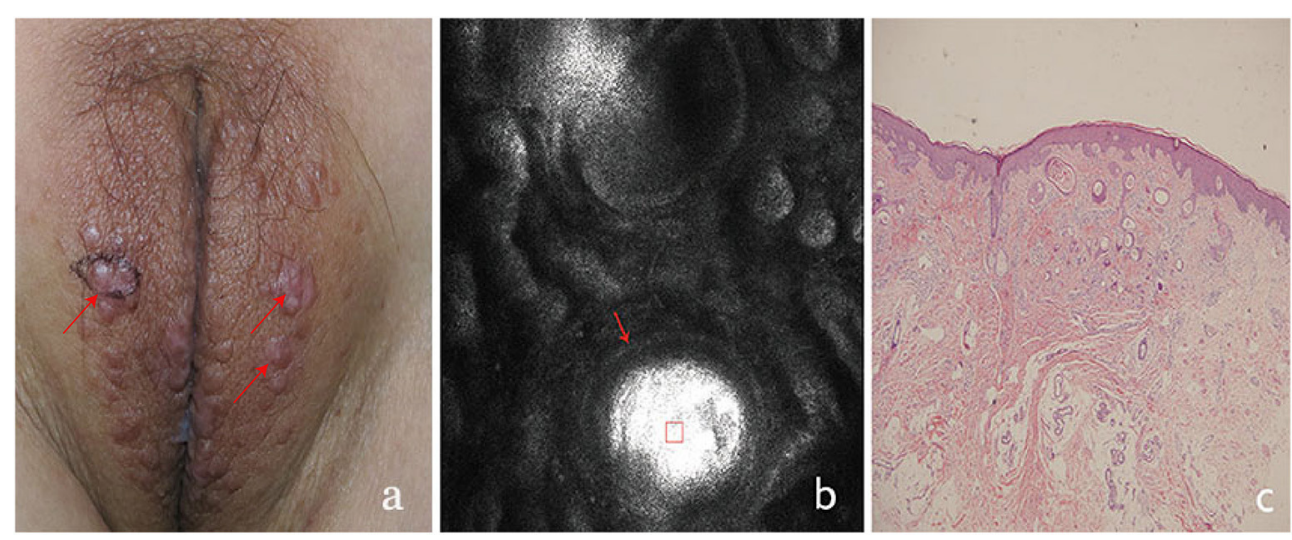

FIGURE 2 | The clinical presentation, RCM findings and histology of vulva syringoma (case 2). (a), the clinical image of case 2, (b), the RCM image findings; (c), the histological findings. The presence of round to oval high refractive, and relatively monomorphous mass of varing sizes (the red square) was present in the superficial dermis, with 1-2 layers of surrounding light-dark line structure (red arrowhead) in (b). 

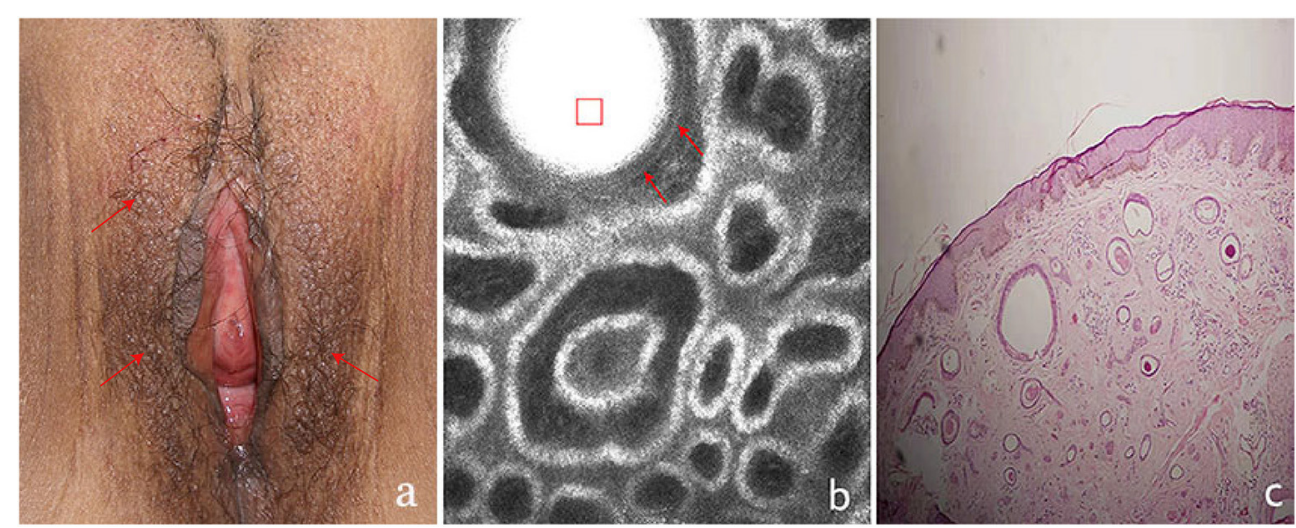

FIGURE 3 | The clinical presentation, RCM findings and histology of vulva syringoma (case 3). (a), the clinical image of case 3, (b), the RCM image findings; (c), the histological findings. The presence of round to oval high refractive, and relatively monomorphous mass of varing sizes (the red square)was present in the upper dermis, with 1-2 layers of surrounding light-dark line structures (red arrowhead)in (b).
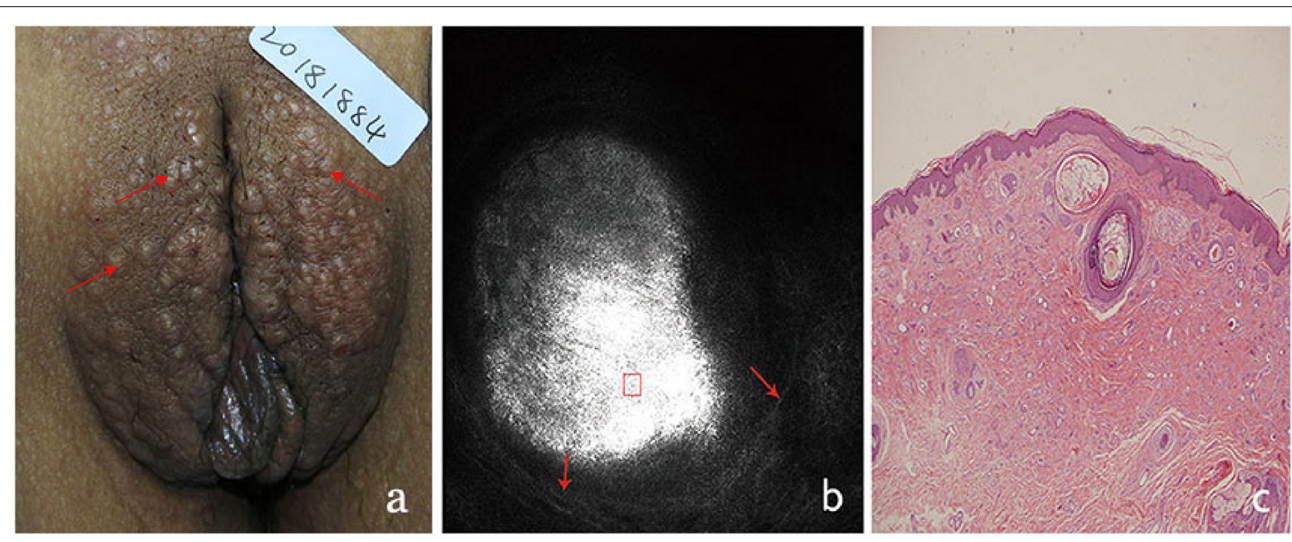

FIGURE 4 | The clinical presentation, RCM findings and histology of vulva syringoma (case 4). (a), the clinical image of case 4, (b), the RCM image findings; (c), the histological findings.

TABLE 1 | The age and medical history of the patients enrolled in the study.

\begin{tabular}{lcccc}
\hline $\begin{array}{l}\text { Case } \\
\text { number }\end{array}$ & Age & $\begin{array}{c}\text { Medical history } \\
\text { (month) }\end{array}$ & $\begin{array}{c}\text { RCM imaging } \\
\text { (+ or }- \text { ) }\end{array}$ & $\begin{array}{c}\text { Histology results } \\
\text { (+ or }- \text { ) }\end{array}$ \\
\hline 1 & 23 & 12 & + & + \\
2 & 32 & 16 & + & + \\
3 & 44 & 20 & + & + \\
4 & 21 & 30 & + & + \\
5 & 32 & 23 & + & + \\
5 & 34 & 14 & + & + \\
7 & 25 & 11 & + & + \\
8 & 27 & 17 & + & + \\
9 & 28 & 9 & + & $+($ milia) \\
10 & 31 & 13 & $-($ milia) & + \\
11 & 39 & 22 & & + \\
\hline Average & 30.5 & 17 & & + \\
\hline
\end{tabular}

+ with significant changes of syringoma in RCM or histology images. - other changes. by RCM were very similar. There were no significant cellular changes in superficial epidermis, and the keratinocytes appeared regular and small, and the architectural pattern of the honeycomb was similar with the peri-lesional normal skin. Deeper level imaging showed brighter keratinocytes in the basal cell layer in lesional zone and the melanin contents in the basal cell layer were also increased compared with the adjacent normal skin.

The most characteristic changes were observed in the upper and middle dermis. Evenly distributed varying sizes of round to oval structures show high refractive mass with surrounding lightdark lines (Figures $\mathbf{1 b}, \mathbf{2 b}, \mathbf{3 b}, \mathbf{4 b}$ ). In comparison, there was just mild to moderate refracted collagen in upper and middle dermis in adjacent non-lesional areas.

The definitive histological diagnoses were all confirmed by biopsy finding in all cases studied, which show classic findings of syringoma with superficial to mid-dermal nests of eccrine ducts and tadpole-like structures embedded in fibrous stroma. clear cell changes of epithelial cells were occasional present. The ductal lumina are filled with an amorphous, periodic 

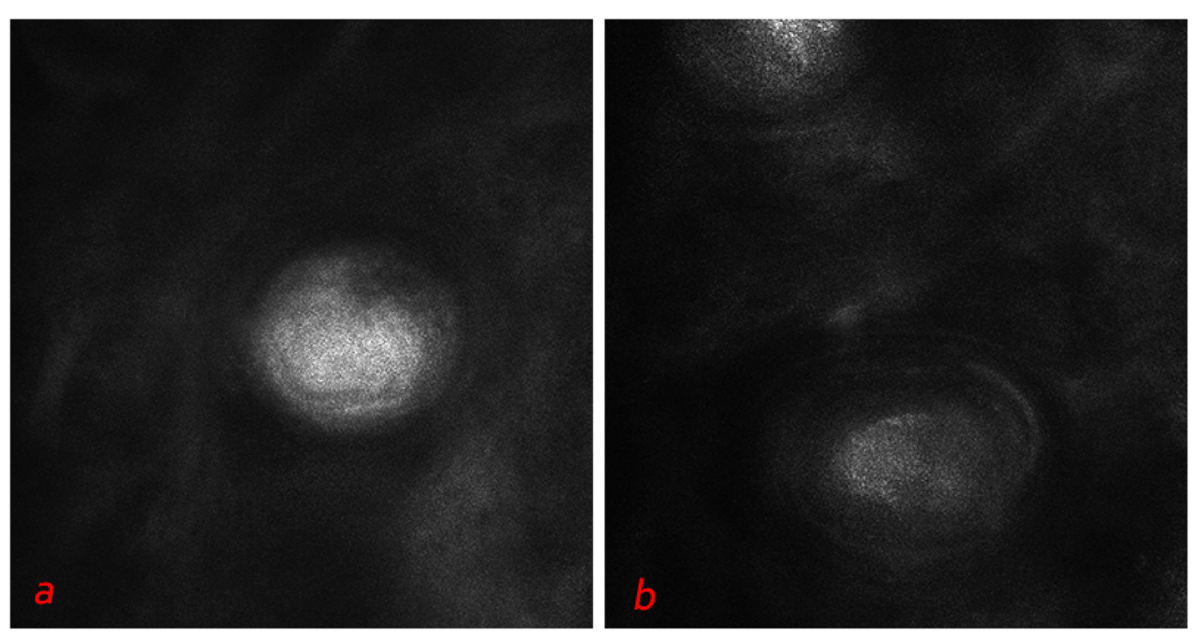

FIGURE 5 | Deeper level imaging of syringoma of vulva area $(\mathbf{a}, \mathbf{b})$ showed that the refraction of the round structures tends to be slightly decreased as they go deep.

acid-Schiff-positive material (Figures 1c, 2c, 3c, 4c), Deeper level imaging showed that the refraction of the round structures tends to be slightly decreased as they go deep (Figures $\mathbf{5 a}, \mathbf{b}$ ).

\section{DISCUSSION}

Syringoma occurs more frequently in adult female with distinct histopathologic features $(1,2)$. In cases with characteristic clinical presentation, syringoma can be easily diagnosed clinically. For lesions on unusual sites such as the genital area, the clinical manifestation was usually atypical. They need to be confirmed by biopsy and histological diagnosis. However, the invasive biopsy procedure would reduce the compliance of the patients, and a non-invasive method for the diagnosis and differential diagnosis of syringoma on vulva sites is definitely sought after by various medical professionals.

The application of dermoscopy imaging in diagnosis of syringoma has been reported and dermoscopy may contribute to the imaging diagnosis of syringoma by revealing multiple bright cystic enlargements in dermis (9). While comparing with dermoscopy, RCM provides more details of those lesions studied with more sensitivity and specificity.

The RCM is also non-invasive and dynamically shows the cellular-level morphology in human skin in vivo $(7,10)$. Imaging is based on the detection of single back scattered photons from the optical section and contrast due to the relative variations in refractive indices and sizes of organelles and micro-structures (7). The cellular changes of the lesion in epidermis and superficial dermis could be imaged and compared with that of the adjacent normal skin. The RCM had been widely used in the screening of skin tumors, such as melanoma $(10,11)$, basal cell carcinoma (12), and actinic keratosis (13). In recent years, the RCM was reported to be useful in the diagnosis, differential diagnosis, and follow-up of pigmented and inflammatory skin disorders $(14,15)$ with high sensitivity and specificity.

The typical changes of syringoma are in mostly confined to superficial and mid-dermis, which is in the imaging depth of RCM. And we found the presence of round to oval, high refractile, and relatively evenly distributed substances of varying sizes in the superficial and mid-dermis with surrounding lightdark line structures based on RCM imaging to be characteristic for synringomas, which were confirmed by histology results. The well-demarcated high refractile substances noted in confocal image correlate with the amorphous, periodic acid-Schiffpositive material in the ductal lumina in histological findings, while the surrounding 1-2 layers of light-dark line structures seen in confocal images correlate with the lining epithelial cells and myoepithelial cells seen in histological images.

Vulva areas can be affected by many papular skin lesions, which should be differentiated from syringoma, those entities include milia, Fox-Fordyce disease, epidermal cysts, lichen planus, and lichen simplex chronicus $(9,16,17)$. The confocal images of milia showed relatively high refractile, but unevenly distributed keratin in superficial dermis (18), which is more superficial compared with syringoma in confocal image findings. The major difference between syringoma and milia in confocal images is the "evenly" refractile substance in dermis and the presence of surrounding 1-2 layers of light and dark lines noted in the former and not present in the latter (18). Our observations are all confirmed with the histological findings. As to other cutaneous adnexal tumors, the RCM could also detect the changes around the follicles and cellular changes in epidermis and superfical dermis, which is useful in the screening and differential diagnosis of those lesions.

A recent case report of syringoma considered the dark areas surrounded by rims of epithelia as the characteristic of syringoma based on RCM findings (19). They interpreted the encircling hollow dark areas as lumina. However, in our study, we identified the round to oval, high refractile, and relatively monomorphous substances of varying sizes in the superficial and middle dermis to represent lumina. And future studies with a larger sample size coupled with histological analysis is needed to confirm our findings.

Our preliminary study showed the novel features of syringoma in RCM imaging, which could be useful as a non-invasive "in 
vivo" tool in the screening and differential diagnosis of suspicious vulva syringomatous lesions. The appropriate clinical application of RCM or other skin imaging methods will potentially reduce the invasive biopsy procedure and related medical cost, and in the end benefit the patients.

\section{DATA AVAILABILITY STATEMENT}

The raw data supporting the conclusions of this article will be made available by the authors, without undue reservation.

\section{ETHICS STATEMENT}

The studies involving human participants were reviewed and approved by The ethic committe of Shandong Provincial Hospital for Skin Diseases. The patients/participants provided their written informed consent to participate in this study.

\section{REFERENCES}

1. Ghanadan A, Khosravi M. Cutaneous syringoma: a clinicopathologic study of 34 new cases and review of the literature. Indian J Dermatol. (2013) 58:326. doi: 10.4103/0019-5154.113956

2. Williams K, Shinkai K. Evaluation and management of the patient with multiple syringomas: a systematic review of the literature. $J$ Am Acad Dermatol. (2016) 74:1234-40. doi: 10.1016/j.jaad.2015. 12.006

3. Ciarloni L, Frouin E, Bodin F, Cribier B. Syringoma: a clinicopathological study of 244 cases. Ann Dermatol Venereol. (2016) 143:5218. doi: 10.1016/j.annder.2015.06.025

4. Friedman S, Butler D. Syringoma presenting as milia. J Am Acad Dermatol. (1987) 16:310-14. doi: 10.1016/S0190-9622(87)70041-3

5. Lau J, Haber RM. Familial eruptive syringomas: case report and review of the literature. J Cutan Med Surg. (2013) 17:84-8. doi: 10.2310/7750.2012. 12027

6. Soler-Carrillo J, Estrach T, Mascaró JM. Eruptive syringoma: 27 new cases and review of the literature. J Eur Acad Dermatol Venereol. (2001) 15:2426. doi: 10.1046/j.1468-3083.2001.00235.x

7. Rajadhyaksha M, Marghoob A, Rossi A, Halpern AC, Nehal KS. Reflectance confocal microscopy of skin in vivo: from bench to bedside. Lasers Surg Med. (2017) 49:7-19. doi: 10.1002/1sm.22600

8. Liu H, Lin Y, Nie X, Chen S, Chen X, Shi B, et al. Histological classification of melasma with reflectance confocal microscopy: a pilot study in Chinese patients. Skin Res Technol. (2011) 17:398-403 doi: 10.1111/j.1600-0846.2011.00517.x

9. Corazza M, Borghi A, Minghetti S, Ferron P, Virgili A. Dermoscopy of isolated syringoma of the vulva. J Am Acad Dermatol. (2017) 76:S37S9. doi: 10.1016/j.jaad.2016.06.009

10. Farnetani F, Manfredini M, Longhitano S, Chester J, Shaniko K, Cinotti E, et al. Morphological classification of melanoma metastasis with reflectance confocal microscopy. J Eur Acad Dermatol Venereol. (2019) 33:67685. doi: $10.1111 /$ jdv.15329

11. Uribe P, Collgros H, Scolyer RA, Menzies SW, Guitera P. In vivo reflectance confocal microscopy for the diagnosis of melanoma and melanotic macules of the lip. JAMA Dermatol. (2017) 153:882-91. doi: 10.1001/jamadermatol.2017.0504

\section{AUTHOR CONTRIBUTIONS}

LF, YL, and LW collected the cases and che clnical and RCM data. HC collected the histology data. MG and HL designed the study and prepared the manuscript. HY revised the manuscript. All authors contributed to the article and approved the submitted version.

\section{FUNDING}

This work was supported by national key scientific instruments and equipment development program of China (2016YFF0101606), Science and Technology Department of Shandong province (2018GSF118030), Shandong Medical, Health Science and Technology Development Plan Project (2018WSA18036) annd clinical research training program of Shanndong Cancer Hospital (2020).

12. Couzan C, Cinotti E, Labeille B, Vercherin P, Rubegni P, Cambazard F, et al. Reflectance confocal microscopy identification of subclinical basal cell carcinomas during and after vismodegib treatment. J Eur Acad Dermatol Venereol. (2018) 32:763-7. doi: 10.1111/jdv.14650

13. Peppelman M, Nguyen KP, Hoogedoorn L, van Erp PE, Gerritsen MJ. Reflectance confocal microscopy: non-invasive distinction between actinic keratosis and squamous cell carcinoma. J Eur Acad Dermatol Venereol. (2015) 29:1302-9. doi: 10.1111/jdv.12806

14. Ardigo M, Agozzino M, Franceschini C, Lacarrubba F. Reflectance confocalmicroscopy algorithms for inflammatory and hair diseases. Dermatol Clin. (2016) 34:487-96. doi: 10.1016/j.det.2016.05.011

15. Cinotti E, Perrot JL, Labeille B, Cambazard F. Reflectance confocal microscopy for cutaneous infections and infestations. J Eur Acad Dermatol Venereol. (2016) 30:754-63. doi: 10.1111/jdv.13254

16. Heller DS. Benign tumors and tumor-like lesions of the vulva. Clin Obstet Gynecol. (2015) 58:526-35. doi: 10.1097/GRF.0000000000000133

17. Naveen KN, Pai VV, Sori T. Syringoma masquerading as steatocystoma multiplex. Indian J Dermatol Venereol Leprol. (2012) 78:365-6. doi: 10.4103/0378-6323.95458

18. Gao M, Lin Y, Wang L, Yu Y, Zhou Y, Chen S, et al. The differential diagnosis of syringoma and milia based on the imaging of reflectance confocal microscopy. Skin Res Technol. (2020) 26:951-3. doi: 10.1111/srt.12879

19. Jiménez MR, Rocchetto H, Ferreira PS, Sangueza M, Lourenço SV, Nico MMS. Evaluation of syringomas by in vivo reflectance confocal microscopy: a report of two cases. Am J Dermatopathol. (2017) 39:845-8. doi: 10.1097/DAD.0000000000000751

Conflict of Interest: The authors declare that the research was conducted in the absence of any commercial or financial relationships that could be construed as a potential conflict of interest.

Copyright (C) 2021 Feng, Lin, Wang, Chen, Gao, Liu and Yang. This is an open-access article distributed under the terms of the Creative Commons Attribution License (CC $B Y)$. The use, distribution or reproduction in other forums is permitted, provided the original author(s) and the copyright owner(s) are credited and that the original publication in this journal is cited, in accordance with accepted academic practice. No use, distribution or reproduction is permitted which does not comply with these terms. 